\title{
Using Neuroscience, Contemporary methods and Traditional Wisdom in Building Well-being in Long Term Unemployed Youth
}

James Ryan, Sam Okoth and Lisa Keegan

Advances in Integrative Medicine, 2019-05-01, Volume 6, Pages S40-S41, Copyright @ 2019

Overview: The Empowering Youth to Thrive (EYTT) program seeks to answer the question whether the combination of Neuroscience, Contemporary Methodologies and Traditional Wisdom can improve the wellbeing of long-term unemployed youth enough to help them move into earning or learning.

Rationale: Long-term unemployed youth have traditionally been a difficult group to shift toward pro-social/wellbeing habits. Using neuroscience, especially the Neurosequential Model of Therapeutics, as an underlying scientific basis for helping and healing young people, EYTT explored layering contemporary and traditional modalities on top of the understandings of neuroscience.

EYTT has shown that using Contemporary Methods, such as Art Therapy, Crosslateral movements, Fitness, Mindfulness and Social \& Emotional Learning, and Traditional Wisdom elements such as stories, mythology, social protocols, Rites of Passage and ceremony/ritual can successfully shape and form youth in the development of pro-social and wellbeing habits.

Objectives: Participants will: gain an understanding of innovative ways of using Neuroscience; hear and learn why stories are essential for human wellbeing and change; take away practical ideas from Traditional Wisdom that work across the three levels of social, ceremonial and spiritual; and explore creative ways of measuring outcomes in youth programs.

Session Summary: James Ryan will present the EYTT project and outline the Neuroscience, Contemporary Methodologies, and Traditional Wisdom components. Lisa Keegan will explain the research methods and findings, and interesting program statistics. Sam Okoth will guide practical Traditional Wisdom elements including M'bas, Drumming and Way of Circle, while demonstrating positive brain changes using EEG headsets. 